PROCEEDINGS OF THE

AMERICAN MATHEMATICAL SOCIETY

Volume 125, Number 1, January 1997, Pages 35-39

S 0002-9939(97)03823-9

\title{
q-ANALOGUE TRIANGULAR NUMBERS AND DISTANCE GEOMETRY
}

\author{
KENNETH B. STOLARSKY
}

(Communicated by Jeffry N. Kahn)

\begin{abstract}
The so-called " $q$-identities" play a major role in classical combinatorics. Most of them can be viewed as arising somehow in the context of hypergeometric series. Here we present a "sum of squares" identity involving $q$-analogues of the triangular numbers that, by contrast, arises in the context of distance geometry.
\end{abstract}

\section{§1. INTRODUCTION}

Our purpose is to establish the following $q$-identity, and explain its relation to distance geometry. For integers $n \geq 1$ define

$$
P_{n}(q)=n^{2} q^{2(n-1)}-\sum_{k=1}^{n-1} k q^{2(k-1)}
$$

and

$$
S_{n}(q)=\frac{\sqrt{P_{n}}(q)}{n}+\sum_{m=1}^{n-1} \frac{\sqrt{P_{m}}(q)}{m(m+1)} .
$$

Set $S_{0}(q)=0$.

Theorem. $\sum_{k=0}^{n}\left(S_{n}(q)-S_{k}(q)\right)^{2}=n q^{2(n-1)}$.

For $q<1$ and $q$ sufficiently close to 1 , this involves only real numbers.

The $n$th triangular number $P_{n}$ is

$$
P_{n}=\sum_{k=1}^{n} k=n(n+1) / 2 ;
$$

two familiar properties of triangular numbers are

$$
n^{2}=P_{n}+P_{n-1} \quad \text { and } \quad \sum_{n=1}^{\infty} \frac{1}{P_{n}}=2 .
$$

Received by the editors June 29, 1995.

1991 Mathematics Subject Classification. Primary 05A19, 05A30, 51K05; Secondary 11B65.

Key words and phrases. Distance geometry, $q$-identity, $q$-analogue triangular numbers, triangular numbers. 
The first immediately shows that $P_{n}(1)=P_{n}$, so $P_{n}(q)$ is a $q$-analogue of $P_{n}$, while the sum in the definition of $S_{n}(q)$ is reminiscent of the second property. Simple estimates show that all the zeros of $P_{n}(q)$ lie strictly inside the unit circle.

The main connection of the identity with distance geometry is given in $\S 3$. Also, the proof of the identity in $\S 2$ below involves an incidental generalization of the familiar parallelogram law (replace the $a_{i}$ by vectors, and interpret $a_{i} a_{j}$ as the inner product $\left(a_{i}, a_{j}\right)$; then the case $n=2$ of the Lemma is the parallelogram law).

\section{$\S 2$. THE PROOF}

For $n \geq 1$ define

$$
L_{n}\left(a_{1}, \ldots, a_{n}\right)=\frac{a_{n}}{n}+\sum_{i=1}^{n-1} \frac{a_{i}}{i(i+1)}
$$

and set $L_{0}=0$.

\section{Lemma.}

$$
\begin{aligned}
F\left(a_{1}, \ldots, a_{n}\right) & :=\sum_{k=0}^{n}\left[L_{n}\left(a_{1}, \ldots, a_{n}\right)-L_{k}\left(a_{1}, \ldots, a_{k}\right)\right]^{2} \\
& =L_{n}\left(a_{1}^{2}, a_{2}^{2}, \ldots, a_{n}^{2}\right) .
\end{aligned}
$$

Proof. Let

$$
\phi(t, j)= \begin{cases}1 /(j(j+1)), & j<t \\ 1 / t, & j=t \\ 0, & j>t\end{cases}
$$

Then

$$
F_{i j}:=\frac{\partial^{2} F}{\partial a_{i} \partial a_{j}}=2 \sum_{k=0}^{n}[\phi(n, j)-\phi(k, j)][\phi(n, i)-\phi(k, i)] .
$$

If $k>\min (i, j)$, then one of the two factors above is 0 . Hence

$$
F_{i j}=2 \sum_{k=0}^{\min (i, j)}[\phi(n, j)-\phi(k, j)][\phi(n, i)-\phi(k, i)] .
$$

First, say $i<j<n$. Then

$$
F_{i j}=\left(2 \sum_{k=0}^{i-1}\right)+\frac{2}{j(j+1)}\left[\frac{1}{i(i+1)}-\frac{1}{i}\right]
$$

where the sum to $(i-1)$ is

$$
2 i \frac{1}{i(i+1)} \frac{1}{j(j+1)} .
$$

Secondly, say $i<j=n$. Then

$$
F_{i j}=\left(2 \sum_{k=0}^{i-1}\right)+\frac{2}{n}\left[\frac{1}{i(i+1)}-\frac{1}{i}\right]
$$


where the sum to $(i-1)$ is $2 /(n(i+1))$. In either case $F_{i j}=0$, so $F_{i j}$ has no "mixed terms" $a_{i} a_{j}$ with $i \neq j$. Next, say $i=j<n$. Then

$$
F_{i j}=\left(2 \sum_{k=0}^{i-1}\right)+2\left[\frac{1}{i(i+1)}-\frac{1}{i}\right]\left[\frac{1}{i(i+1)}-\frac{1}{i}\right]
$$

where the sum to $(i-1)$ is

$$
\frac{2 i}{[i(i+1)]^{2}} \text {. }
$$

Upon dividing by 2 ! we find that the coefficient of $a_{i}^{2}$ is $1 /(i(i+1))$. Finally, the coefficient of $a_{n}^{2}$ is $1 / n$ by direct inspection. This proves the Lemma.

We can now prove the Theorem. By the Lemma, the sum of the squares is

$$
\sum=\frac{P_{n}(q)}{n}+\sum_{k=1}^{n-1} \frac{P_{m}(q)}{k(k+1)} .
$$

Clearly this polynomial in $q$ has $n q^{2(n-1)}$ as its highest order term. Its constant term is

$$
\frac{1}{2}-\left(\sum_{k=2}^{n-1} \frac{1}{k(k+1)}+\frac{1}{n}\right)=\frac{1}{2}-\left(1-\frac{1}{2}\right)=0 .
$$

In general, the coefficient of $q^{2 m}$ is

$$
\begin{aligned}
& \frac{(m+1)^{2}}{(m+1)(m+2)}-\left(\sum_{k=m+2}^{n-1} \frac{m+1}{k(k+1)}+\frac{m+1}{n}\right) \\
& =(m+1)\left(\frac{1}{m+2}-\left(\sum_{k=m+2}^{n-1} \frac{1}{k(k+1)}+\frac{1}{n}\right)\right)
\end{aligned}
$$

and (by partial fractions, for example) this is easily seen to be 0 .

Remark. If the exponent 2 on the left-hand summand of the Lemma is replaced by 1 , the sum becomes simply $a_{n}$. Essentially the same proof works (with rather simpler details). Now, by Hölder's inequality,

$$
f(n, q, \lambda):=\left(\frac{1}{n} \sum\left|S_{n}(q)-S_{k}(q)\right|^{\lambda}\right)^{1 / \lambda} \leq q^{n-1}
$$

for $1 \leq \lambda \leq 2$. Here $f(n, 1, \lambda)$ seems to be decreasing (slowly) in $n$. Set

$$
g(\lambda)=\liminf _{n \rightarrow \infty} f(n, 1, \lambda), \quad 1 \leq \lambda \leq 2 .
$$

From the above it is not hard to see that

$$
\frac{1}{\sqrt{2}}=g(1) \leq g(\lambda) \leq g(2)=1, \quad 1 \leq \lambda \leq 2,
$$

and from a familiar property of power means we see that $g(\lambda)$ is non-decreasing. Probably liminf may be replaced by lim, but the author has not been able to identify $g(\lambda)$. 


\section{§3. Connection with Distance Geometry}

A fundamental question in distance geometry is the following: given a set of points $S$ and an assignment of a distance to every pair of distinct points in $S$, can one find in a given metric space $M$ a set of points $S^{*}$ in one-to-one correspondence with $S$ and with corresponding distances equal? This, of course, is the problem of isometric embedding. For example, if $|S|=4$ and every distance is 1 , this can be done if $M=E^{3}$ but not if $M=E^{2}$. For a definitive treatise on distance geometry see $[\mathrm{B}]$.

We now restrict ourselves to a quite specific question. Given a curve in a metric space $M$, does it contain a sequence of distinct points $x_{0}, x_{1}, x_{2}, x_{3}, \ldots$ (in that order, say) such that the distance from $x_{n}$ to the previous points (i.e., to the $x_{i}$ with $0 \leq i \leq n-1)$ is on average always 1? For the Euclidean line the sequence of initial segments of the harmonic series, namely

$$
0, \quad 1, \quad 1+\frac{1}{2}, \quad 1+\frac{1}{2}+\frac{1}{3}, \quad 1+\frac{1}{2}+\frac{1}{3}+\frac{1}{4}, \ldots,
$$

is quickly seen to have this property. Next, fix $q<1$. Is there such a sequence for which the average distance from $x_{n}$ to the previous $x_{i}$ is $q^{n-1}$ (this is the $q$-analogue of the previous question)? Here $x_{0}=0$ and

$$
x_{n}=q^{n-1}+\sum_{m=2}^{n} \frac{q^{m-2}}{m}
$$

is quickly seen to have this property. Moreover, the $x_{n}$ converge to a limit, namely

$$
x_{\infty}=-[q+\log (1-q)] / q^{2} \text {. }
$$

(The monotone dependence of $x_{\infty}$ on $q$ is equivalent to the inequality $-\log (1-q)<$ $q+q^{2} /(2(1-q))$.) The problem is more interesting, however, if we replace average distance by root-mean-square distance. In this case, for $q<1$, no such sequence can be infinite, but for $q$ sufficiently close to 1 such a sequence may have an arbitrarily large number of terms. To see this, simply note that since the $x_{k}$ are an increasing sequence that is determined sequentially, they must coincide with the $S_{n}(q)$ of the Theorem! Now

$$
S_{n+1}(1)-S_{n}(1)=\frac{-\sqrt{P_{n}}}{n+1}+\frac{\sqrt{P_{n+1}}}{n+1}>0,
$$

so the $S_{n}(q)$ form an increasing sequence when $q$ is close enough to 1 . Moreover, for $q<1$ fixed but $n$ large,

$$
\begin{aligned}
P_{n}(q) & \leq n^{2} q^{2(n-1)}-\sum_{k=1}^{[3 n / 4]} k q^{2(k-1)} \\
& \leq n^{2} q^{2(n-1)}-q^{[3 n / 2]} \sum_{k=1}^{[3 n / 4]} k \\
& \leq n^{2} q^{2(n-1)}-\frac{1}{8} n^{2} q^{[3 n / 4]}<0 .
\end{aligned}
$$

Hence the process always terminates.

Remarks. This geometric problem was suggested by the 1964 theorem of Gross $[\mathrm{G}, \mathrm{C}-\mathrm{F}-\mathrm{G}]$ in game theory that has found a home in distance geometry. For a 
survey and further references see [C-M-Y]. For a survey of $q$-analogues, see $[\mathrm{A}]$. An application of isometric embedding to a sum of distances problem is given in [S].

\section{REFERENCES}

[A] Andrews, G. E., q-Series: their development and application in analysis, number theory, combinatorics, physics, and computer algebra, Regional Conference Series in Math. 66, Amer. Math. Soc. (1986). MR 88b:11063

[B] Blumenthal, L. M., Theory and Applications of Distance Geometry, Chelsea, New York, 1970. MR 42:3678

[C-M-Y] Cleary, J., Morris, S. A., and Yost, D., Numerical geometry-numbers for shapes, Amer. Math. Monthly 93 (1986), 260-275. MR 87h:51043

[C-F-G] Croft, H. T., Falconer, K. J., and Guy, Richard K., Unsolved Problems in Geometry, Springer-Verlag, New York, 1991. MR 92c:52001

[G] Gross, O., The rendezvous value of a metric space, Advances in Game Theory, Ann. Math. Studies, vol. 52, Princeton University, Princeton, 1964, pp. 49-53. MR 28:5841

[S] Stolarsky, K. B., Sums of distances between points on a sphere, Proc. Amer. Math. Soc. 35 (1972), 547-549. MR 46:2555

Department of Mathematics, University of Illinois, 1409 W. Green St., Urbana, IlliNOIS 61801

E-mail address: stolarsk@math.uiuc.edu 